\title{
Model Predictive Control Applied to Constraint Handling in Active Noise and Vibration Control
}

\author{
Adrian G. Wills, Dale Bates, Student Member, IEEE, Andrew J. Fleming, Member, IEEE, \\ Brett Ninness, Member, IEEE, and S. O. Reza Moheimani, Senior Member, IEEE
}

\begin{abstract}
The difficulties imposed by actuator limitations in a range of active vibration and noise control problems are well recognized. This paper proposes and examines a new approach of employing model predictive control (MPC). MPC permits limitations on allowable control action to be explicitly included in the computation of an optimal control action. Such techniques have been widely and successfully applied in many other areas. However, due to the relatively high computational requirements of MPC, existing applications have been limited to systems with slow dynamics. This paper illustrates that MPC can be implemented on inexpensive hardware at high sampling rates using traditional online quadratic programming methods for nontrivial models and with significant control performance dividends.
\end{abstract}

Index Terms-Active noise and vibration control, active structures, model predictive control (MPC), piezoelectric actuators.

\section{INTRODUCTION}

A CTIVE control of sound and vibration [16], [35] has shown promise in a variety of applications ranging from consumer and automotive products to military and aerospace systems. In addition to increasing performance, the primary motivation is to reduce the weight and volume requirements of traditional passive treatments such as mechanical tuned-mass absorbers [24] and acoustic Helmholtz resonators [33]. At low frequencies, below $500 \mathrm{~Hz}$, such techniques can become prohibitively large and impractical.

In light of this, a large number of techniques for active noise and vibration control have been proposed in the literature and applications of the same are found in robotics [9], [30] maritime technology [10], and airfoil control [23]. For mechanical systems, a commonly applied method is positive position feedback (PPF) control as proposed by Caughey and co-workers [11], [14], [18], [32]. Other methods, including optimal approaches such as $H_{\infty}$ [2] and minimax Linear Quadratic Gaussian (LQG) [37], have also been prominent but are less frequently applied in practice.

One of the key issues limiting the industrial application of high-performance noise and vibration control technology is the

Manuscript received June 23, 2006; revised January 16, 2007. Manuscript received in final form March 6, 2007. Recommended by Associate Editor G. Stewart. This work was supported in part by the Australian Research Council and by the Centre for Complex Dynamic Systems and Control.

The authors are with the School of Electrical Engineering and Computer Science, University of Newcastle, Callaghan, NSW, Australia (e-mail: adrian. wills@newcastle.edu.au).

Color versions of one or more of the figures in this paper are available online at http://ieeexplore.ieee.org.

Digital Object Identifier 10.1109/TCST.2007.903062 problem of actuator saturation. Transducer limitations are usually handled by upgrading the sensors and actuators until the dynamic range significantly exceeds the expected signal range. The consequences of this approach are extremely undesirable.

First, an equivalent transducer with greater dynamic range has decreased signal-to-noise level, and for sampled systems, less resolution. In addition, an over-specified transducer may be physically larger, heavier, more costly and consume more power than moderately specified transducers.

The second, and more important consequence, is the question of reliability and performance. Regardless of the actuator dynamic range, it is almost always possible to imagine scenarios where an unusually large excitation could result in actuator saturation. In these circumstances, the control system should provide the greatest immunity to disturbance, while in practice some controllers drive the actuators hard into saturation, which effectively opens the control loop, and consequently, degrades regulatory performance. Worse still, the controller may become unstable and itself contribute to increased vibration and mechanical failure. In the high-reliability applications targeted by active noise and vibration control, for example aerospace structural control [23], all of the potential benefits may amount to nothing if the system does not remain functional and reliable during all feasible environmental conditions.

In addition to the over-specification of transducer requirements, another standard technique for dealing with actuator saturation is to augment the controller with a saturation block [42]. This technique is not suitable when considering lightly-damped resonant systems as the broad-spectrum saturation event can excite a large number of modes. A final unattractive technique is to simply detune the controller thereby reducing the possibility of actuator saturation [19], [22], [36].

In response to these shortcomings, and given a history of successes in other areas for handling hard actuator limits, here we examine the use of model predictive control (MPC) for the purposes of active noise cancellation and vibration control in the presence of hard actuator limits.

While, on the one hand, MPC is related to LQG control and is known to offer good dynamic performance, on the other hand, it also caters for hard actuator limits and other system constraints in a straightforward manner [28]. Such constraints enter MPC as side conditions on an optimization problem, which is solved online and at each control interval to obtain the next control action [7], [8], [17], [27], [34].

While solving this optimization problem is at the heart of MPC and provides it with the ability to easily handle constraints, it also presents a challenge in that this problem can be difficult to 
solve in real time. Certainly, the computational burden increases as sampling rates increase and this has limited the application of MPC to systems with relatively slow dynamics [39]. For example, MPC has been used within the petrochemical industry since the 1970s in the form of supervisory control, where sample times are in the order of minutes, hours, or even days [39].

In order to reduce the computational effort required to solve this problem online, recent work in the area has considered the use of multiparametric programming techniques such as those described in [4], [6], and [41]. This approach has been called explicit-MPC and generates a "lookup table" offline, which is then implemented online in a straightforward and computationally inexpensive manner. Even more recently, specific hardware implementations of explicit-MPC have been considered with very promising results at high sampling rates [25].

The more traditional alternative approach would employ an optimization algorithm such as an active-set or interior-point method in order to solve this problem online.

The choice between using explicit-MPC or more traditional methods depends largely on the particular problem being considered (see, e.g., [25]). A major drawback of the explicit approach is the curse of dimensionality; as the degrees-of-freedom increase the memory requirements and number of entries in the lookup table can grow dramatically.

In light of this, the more traditional MPC approach is used in this paper since the dimension of this problem currently precludes the use of explicit-MPC on the available hardware (see Section IV-A for a discussion).

In particular, we consider a cantilevered beam example, which is representative of many flexible structures, and employ a standard active-set method in order to solve the associated quadratic program online.

This presents a very challenging situation for standard MPC since the beam exhibits a wide range of oscillatory dynamics, from slow $(5 \mathrm{~Hz})$ to fast $(500 \mathrm{~Hz})$, which requires an 18th-order model and a sampling rate of at least $5 \mathrm{kHz}$ for control purposes. Furthermore, in the interests of having a practical implementation, a very inexpensive floating-point hardware platform is used to realize the MPC algorithm.

In light of this, the contributions of this paper are twofold. First, we extend previous results on constraint handling for active structures and show that MPC pays dividends compared with already accepted strategies. In particular, it offers improved dynamic performance in the presence of actuator limitations.

Second, we demonstrate that using an off-the-shelf active-set method, MPC can be applied to systems with relatively broad and complex dynamics (five bending modes between 5 and $500 \mathrm{~Hz}$ using an 18th-order model), using nontrivial prediction horizons (12 steps ahead), while respecting input constraints, using a sampling rate of $5 \mathrm{kHz}$, and with implementation on an inexpensive 200-MHz digital signal processor.

\section{Problem Definition}

Active noise and vibration control problems can generally be represented by the feedback diagram shown in Fig. 1. Commonly encountered mechanical [16], [24] and acoustic plants [35] comprise a large number of lightly damped, close-in-frequency, resonant modes. As higher frequency dynamics are usu-

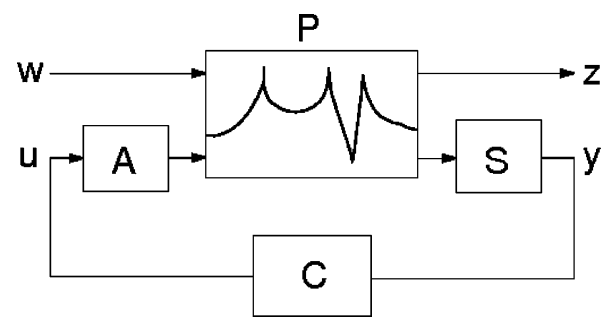

Fig. 1. Typical active noise control scenario.

ally controlled by passive means, the bandwidth of interest in active noise and vibration control is approximately $1 \mathrm{kHz}$. Depending on the system, this may require the control of just a few to many hundreds of modes.

A feedback controller $C(s)$ is designed to regulate the performance variable $z$ in response to external input disturbances $w$. The performance variable $z$ may be a physical quantity such as the acceleration at a point, or represent an abstract quantity such as modal energy or spatial deflection [31].

Examples of measurement and control variables include: force, strain, displacement, torque, moment, acceleration, velocity, angle, and pressure. All of these variables require transducers to convert to and from the physical domain. In Fig. 1, actuating and sensing transducers are denoted by $A$ and $S$, respectively. In addition to their linear dynamics, the actuating and sensing transducers $A$ and $S$ incorporate a number of constraints that correspond to the physical limitations inherent in each device.

Saturation, where a variable is constrained to lie within particular limits, is the most commonly encountered constraint. Examples found in active noise control include: the output voltage and current range of a voltage amplifier, the displacement range of a speaker baffle, the pressure range of a microphone, the depolarization voltage of a piezoelectric transducer and the force limit of an inertial drive. Saturation constraints can represent situations where there exist insufficient resources to develop the required output, or where reproduction of the required output would result in mechanical or electrical failure.

Another less commonly encountered constraint is the slewrate or rate-of-change limit in a physical variable. Voltage feedback amplifiers are slew-rate limited by both internal saturation and current limits when driving capacitive loads. Electromagnetic actuators, such as voice coil speakers, have limited velocity and displacement slew-rate imposed by the maximum available driving current.

In summary, our objective is to derive a control law that minimizes the performance variable $z$ subject to a set of actuator constraints. The synthesis technique should be able to handle wide-band systems with a large number of poles in close proximity to the imaginary axis. In the following, MPC is proposed as a solution and verified experimentally by suppressing vibration in a cantilever beam.

\section{MPC FORMULATION}

As already mentioned, a major attraction of MPC is its ability to handle constraints, which is the main reason for its consideration here. Constraints enter MPC straightforwardly as side 
conditions on the MPC optimization problem. This problem is solved at each control interval in order to compute the next optimal control action. To make this more precise, we define the control action generated by MPC as follows.

Definition III.1: The control action generated by MPC for time $t+1$ given information up to and including time $t$ is denoted by $u_{t+1 \mid t}^{*}$ and is defined as the first element of the sequence

$$
\mathbf{u}_{t}^{*} \triangleq\left\{u_{t+1 \mid t}^{*}, \ldots, u_{t+N \mid t}^{*}\right\}
$$

which is defined as the solution to the optimization problem

$$
\mathbf{u}_{t}^{*}=\arg \min _{\mathbf{u}_{t}} J\left(\mathbf{u}_{t}\right) \quad \text { s.t. } \quad \mathbf{u}_{t} \in \mathbb{U} .
$$

In order to adequately describe the optimization problem in (2), we diverge momentarily to introduce a system model. Specifically, we assume that the system behaves according to the linear time invariant state-space system

$$
\begin{aligned}
x_{t+1} & =A x_{t}+B u_{t}+\omega_{t} \\
y_{t} & =C x_{t}+D u_{t}+e_{t}
\end{aligned}
$$

where $u_{t} \in \mathbf{R}^{m}$ is the input, $y_{t} \in \mathbf{R}^{p}$ is the output, $x_{t} \in \mathbf{R}^{n}$ is the state, and $\omega_{t} \in \mathbf{R}^{n}$ and $e_{t} \in \mathbf{R}^{p}$ are independently and identically distributed (in time) Gaussian stochastic processes with joint distribution

$$
\left[\begin{array}{l}
\omega_{t} \\
e_{t}
\end{array}\right] \sim \mathcal{N}\left\{0,\left[\begin{array}{ll}
W & S \\
S^{T} & E
\end{array}\right]\right\} .
$$

With this in place, the paper considers the following cost function:

$$
J\left(\mathbf{u}_{t}\right)=\left\|\widehat{x}_{t+N+1 \mid t}\right\|_{P}^{2}+\sum_{k=1}^{N}\left\|\widehat{y}_{t+k \mid t}\right\|_{Q}^{2}+\left\|u_{t+k}\right\|_{R}^{2} .
$$

In the previous equation, $\|z\|_{M}^{2} \triangleq z^{T} M z$, where $M$ is assumed to be positive definite and symmetric. The positive integer $N$ is known as the prediction horizon, $\widehat{y}_{t+k \mid t}$ is the predicted output at time $t+k$ given measurements up to and including time $t$, and $\widehat{x}_{t+N+1 \mid t}$ is the predicted state at time $t+N+1$.

In the interest of nominal closed-loop stability it is important to choose the terminal weighting matrix $P$ carefully [7]. As motivation for this, consider the case where there are no constraints in (2), i.e., if $U=\mathbb{R}^{N m}$, then its solution coincides with the well known LQG solution for any prediction horizon $N>0$ when the weighting matrix $P$ is chosen as the positive definite solution to the following discrete algebraic Riccati equation (DARE—see, e.g., [5])

$$
\begin{aligned}
& P=C^{T} Q C+A^{T} P A-K\left(B^{T} P B+R+D^{T} Q D\right) K^{T} \\
& K=-\left(A^{T} P B+C^{T} Q D\right)\left(B^{T} P B+R+D^{T} Q D\right)^{-1} .
\end{aligned}
$$

While this choice for $P$ does not guarantee closed-loop stability when constraints are present, a sufficiently large prediction horizon $N$ combined with this choice does [28].

Clearly, with the choice of $P, N, Q$, and $R$ already determined, in order to use this cost function, we require predictions of the system outputs $\left\{\widehat{y}_{t+1 \mid t}, \ldots, \widehat{y}_{t+N \mid t}\right\}$ and state $\widehat{x}_{t+N+1 \mid t}$. This is typically achieved using a state observer, and while the possibilities for this are many, we propose the use of optimal (in terms of minimum variance) predictions as given by the Kalman Filter (see, e.g., [1])

$$
\begin{aligned}
\widehat{x}_{t+1 \mid t} & =(A-L C) \widehat{x}_{t \mid t-1}+(B-L D) u_{t}+L y_{t} \\
\widehat{x}_{t+k+1 \mid t} & =A \widehat{x}_{t+k \mid t}+B u_{t+k \mid t}, \quad t=1, \ldots, N \\
\widehat{y}_{t+k \mid t} & =C \widehat{x}_{t+k \mid t}+D u_{t+k \mid t}, \quad t=1, \ldots, N .
\end{aligned}
$$

In the previous equation, $L$ is known as the Kalman gain matrix and is given by (again employing the positive definite solution of a DARE)

$$
\begin{aligned}
L & =\left(A X C^{T}+S\right)\left(C X C^{T}+E\right)^{-1} \\
X & =W+A X A^{T}-L\left(C X C^{T}+E\right) L^{T} .
\end{aligned}
$$

It is important to note that the previous predictions depend only on future inputs $u_{t+1 \mid t}, \ldots, u_{t+N \mid t}$, which are free variables. This is essential because it allows a search for the best (according to the cost $J$ ) sequence of future inputs.

The remaining component of the optimization problem (2) are the side conditions or constraints $\mathbf{u}_{t} \in \mathbb{U}$. The types of constraints considered in this paper include linear equality, linear inequality, and simple bounds constraints. Therefore, it suffices to assume that the constraint set $\mathbb{U}$ is represented by

$$
\mathbb{U} \triangleq\left\{\mathbf{u} \in \mathbf{R}^{N p}: A_{e} \mathbf{u}=b_{e}, A_{i} \mathbf{u} \leq b_{i}, b_{\ell} \leq \mathbf{u} \leq b_{u}\right\}
$$

where the previous inequalities are element-wise.

\section{SUGGESTED MPC IMPLEMENTATION}

With computational demand being of great practical interest, it is important to minimize unnecessary overhead for online computations and move certain operations offline. In this section, we suggest one particular implementation of MPC where a distinction is made between online and offline calculations.

Broadly speaking, offline calculations involve objects that infrequently (or never) change, for example, the system matrices $A, B, C, D$, tuning parameters $Q$ and $R$, observer gain matrix $L$, and prediction horizon $N$. On the other hand, online calculations are reserved for objects that do frequently change, such as output measurements $y_{t}$, the state estimate $\widehat{x}_{t+1 \mid t}$, and the optimal input $u_{t+1 \mid t}^{*}$.

More specifically, the following offline procedure is required before control commences.

Procedure 1: Given the plant model $A, B, C, D$ :

1) generate the state observer gain matrix $L$;

2) choose the prediction horizon $N$ and the state and input weighting matrices $Q$ and $R$;

3) calculate $P$ according to (6);

4) construct the constraint set $U$ according to physical limitations and desired operating ranges.

Once the previous procedure is complete, MPC is implemented online according to the following Algorithm. 
Algorithm IV.1 At each time interval $t$, complete the following tasks:

1) apply the previously calculated control move $u_{t \mid t-1}^{*}$ to the system (calculated as $u_{t+1 \mid t}^{*}$ in the previous iteration);

2) measure the system output $y_{t}$;

3 ) estimate the state $\widehat{x}_{t+1 \mid t}$ using the measured outputs and inputs according to the observer (8);

4) compute the next control move $u_{t+1 \mid t}^{*}$ by selecting the first control move from $\mathbf{u}_{t}^{*}$, which is obtained by solving (2).

Computing the next control action (Step 4 in the previous Algorithm) is the most computationally demanding operation involved in implementing MPC. Therefore, the choice of optimization algorithm will, to a large extent, determine the fastest sampling rate possible for the controller and, consequently, restrict the application of MPC to systems of appropriate dynamics. In light of this, Section IV-A discusses one particularly suitable algorithm for solving quadratic programs of the type arising in MPC.

\section{A. Quadratic Programming Solver}

By construction, the optimization problem (2) is in the form of a convex quadratic program. To make this more precise, define a stacked version of the predicted output and terminal state as $\mathbf{y}_{t}$, and denote by $\mathbf{u}_{t}$ a stacked version of future inputs

$$
\mathbf{y}_{t} \triangleq\left[\begin{array}{c}
\widehat{y}_{t+1 \mid t} \\
\vdots \\
\widehat{y}_{t+N \mid t} \\
\widehat{x}_{t+N+1 \mid t}
\end{array}\right], \quad \mathbf{u}_{t} \triangleq\left[\begin{array}{c}
u_{t+1 \mid t} \\
\vdots \\
u_{t+N \mid t}
\end{array}\right] .
$$

From (9) and (10), it then follows that

$$
\mathbf{y}_{t}=\Lambda \widehat{x}_{t+1 \mid t}+\Phi \mathbf{u}_{t}
$$

where

$$
\Lambda \triangleq\left[\begin{array}{l}
C \\
C A \\
C A^{2} \\
\vdots \\
C A^{N-1} \\
A^{N}
\end{array}\right], \Phi \triangleq\left[\begin{array}{lllll}
D & & & & \\
C B & D & & & \\
C A B & C B & D & & \\
\vdots & & & \ddots & \\
C A^{N-2} B & \cdots & \cdots & C B & D \\
A^{N-1} B & \cdots & \cdots & A B & B
\end{array}\right] .
$$

Using these definitions, the cost function $J$ can be expressed as

$$
J\left(\mathbf{u}_{t}\right)=\mathbf{u}_{t}^{T} H \mathbf{u}_{t}+2 \mathbf{u}_{t}^{T} f\left(\widehat{x}_{t+1 \mid t}\right)+c .
$$

Here, $c$ is a constant term that may be ignored and the terms $H$ and $f$ are given by

$$
H \triangleq \Phi^{T} \bar{Q} \Phi+\bar{R}, \quad f\left(\widehat{x}_{t+1 \mid t}\right) \triangleq \Gamma \widehat{x}_{t+1 \mid t}, \quad \Gamma \triangleq \Phi^{T} \bar{Q} \Lambda
$$

with $\bar{Q}$ and $\bar{R}$ being block diagonal matrices given by

$$
\bar{Q} \triangleq\left[\begin{array}{cccc}
Q & & & \\
& \ddots & & \\
& & Q & \\
& & & P
\end{array}\right], \quad \bar{R} \triangleq\left[\begin{array}{llll}
R & & & \\
& R & & \\
& & \ddots & \\
& & & R
\end{array}\right] \text {. }
$$

In light of the previous definitions, (2) can be equivalently stated as

$$
\mathbf{u}_{t}^{*}=\arg \min _{\mathbf{u}_{t}} \mathbf{u}_{t}^{T} H \mathbf{u}_{t}+2 \mathbf{u}_{t}^{T} f\left(\widehat{x}_{t+1 \mid t}\right) \quad \text { s.t. } \mathbf{u}_{t} \in \mathbb{U} .
$$

TABLE I

APPROXIMATE MEMORY REQUIREMENTS FOR EXPLICIT MPC FOR DIFFERENT Horizons $N$. NOTE, THIS IS THE CONTROLLER COMPONENT ONLY, STORAGE OF THE LOOKUP TABLE REQUIRES MORE MEMORY

\begin{tabular}{|c|c|c|c|c|c|}
\hline N & 4 & 6 & 8 & 10 & 12 \\
\hline Regions & 81 & 729 & 6353 & 56761 & $\sim 200000$ \\
\hline Memory (Mbit) & 0.2 & 2.7 & 31 & 345 & $\sim 1460$ \\
\hline
\end{tabular}

TABLE II

MEMORY REQUiREMENTS FOR OBSERVER AND QUADRATIC PROGRAMMING SOLVER ON DSP, WHERE $n$ IS THE NUMBER OF STATES, $p$ IS THE NUMBER OF OUTPUTS, $m$ IS THE NUMBER OF INPUTS, AND $N$ IS THE PREDICTION HORIZON

\begin{tabular}{|c|c|}
\hline Data for & No. of 32 bit words \\
\hline Observer & $n(n+m+p+2)+m+p+3$ \\
\hline QP & $4(N m)^{2}+N m(n+6)+5$ \\
\hline
\end{tabular}

In this form, the optimization problem may be solved using standard quadratic programming routines, or alternatively, using a multiparametric programming approach [3], [6], [15], [41], [44], [46]. Both approaches generate identical solutions, but differ in implementation; the former approach employs a general algorithm to solve the quadratic program online; the latter exploits the fact that the solution to (15) is piecewise-affine [46] in the state (or parameters) $\widehat{x}_{t+1 \mid t}$, and constructs all necessary polytopes and associated control laws offline, which results in a straightforward online implementation (see [44] for details).

While a full quantitative comparison of the two approaches is beyond the scope of this paper, an attempt at using the multiparametric approach quickly identified one of its widely known drawbacks, namely the potentially very demanding memory requirements (see, e.g., [25]). In particular, for the system considered in this paper, Table I shows a portion of the memory requirements for various prediction horizons. Bearing in mind that the hardware has $1 \mathrm{Mb}$ of storage capacity and that $N=$ 12 is the desired prediction horizon for this application (see Section V), Table I indicates that the multiparametric approach is not currently suitable for this application. Therefore, a more traditional online approach was employed (see Table II to compare memory requirements for these two approaches).

Within the class of direct online methods, the two most commonly used algorithms are active-set and interior-point methods, which have numerous suitable implementations. ${ }^{1}$ However, for time-critical online optimization it is often necessary to adapt such tools in order to exploit problem structure.

In light of this, we have implemented an active-set method based on the work of [20], [38], and [40]. While this method requires a positive definite Hessian matrix $H$, which is automatically satisfied, for example, whenever $R$ is positive definite, it does not require a feasible initial point, which greatly simplifies the algorithm. It handles equality, general inequality, and simple bound constraints in a straightforward manner and caters for hot "starting," i.e., where previous solutions are used to initialize the algorithm. Furthermore, it has been refined in the open literature and open source community for some 20 years.

A cursory description of the algorithm is as follows. Starting with the unconstrained solution $\left(-H^{-1} f\right)$, each subsequent iteration adds a violated constraint, if any, to the active-set of con-

\footnotetext{
${ }^{1}$ See http://www.numerical.rl.ac.uk/qp/qp.html.
} 


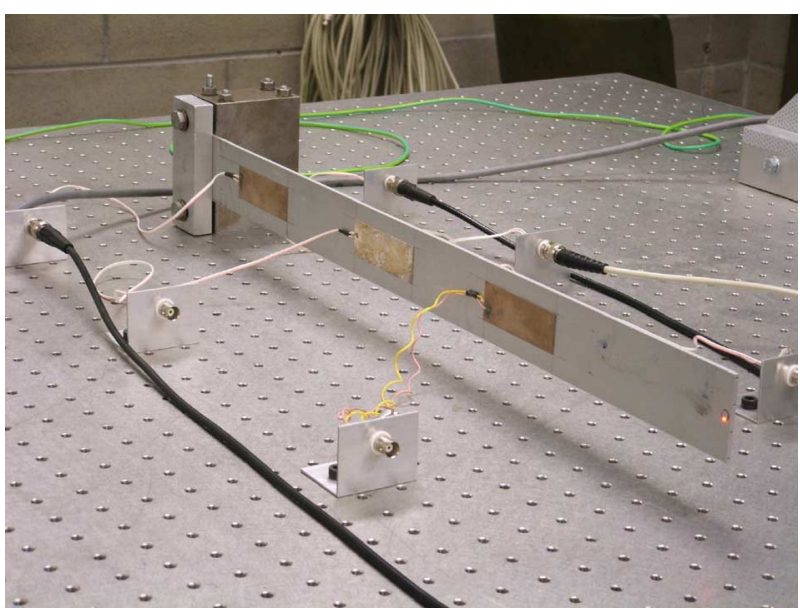

Fig. 2. Experimental apparatus.

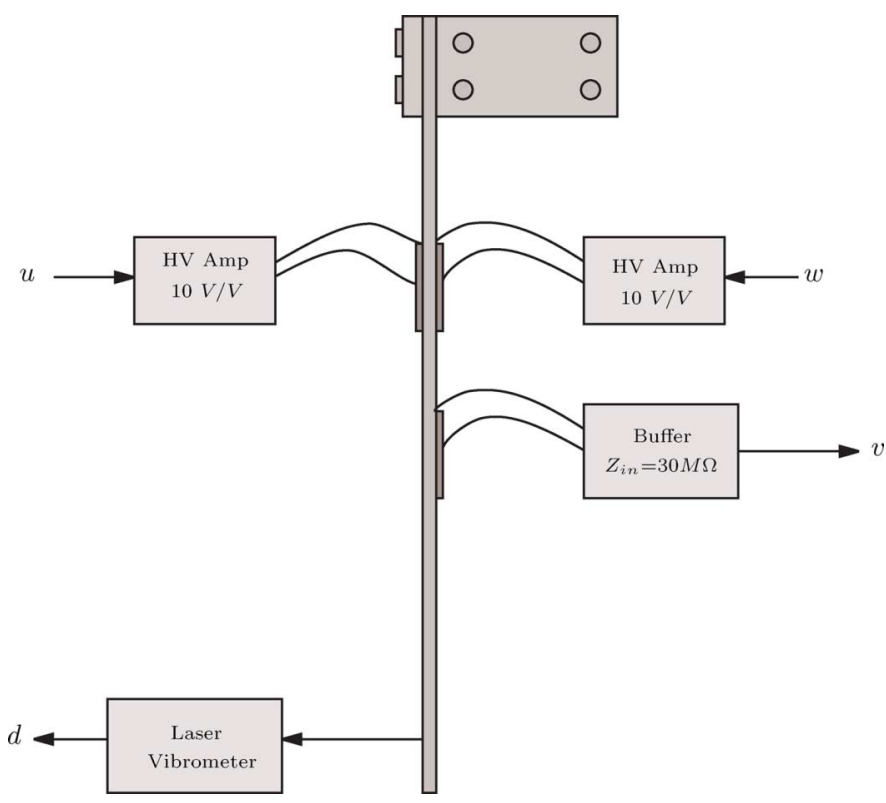

Fig. 3. Plan view schematic of the experimental apparatus.

straints and then solves an equality constrained problem, where the equality constraints are those listed in the active-set. There is also the possibility of dropping an already active constraint if no longer needed (i.e. the associated Lagrange multiplier is negative).

The method maintains two matrices $Z_{a}$ and $R_{a}$ such that $Z_{a} Z_{a}^{T}=H^{-1}$ and $Z_{a}^{T} A_{a}=R_{a}$, where the columns of $A_{a}$ hold the normals to the active constraints and $R_{a}$ is an upper triangular matrix. The implementation uses Givens rotations (see, e.g., [21]) to update the matrices $Z_{a}$ and $R_{a}$ in a numerically robust fashion. If there are no more constraints in violation then the algorithm terminates and the solution is optimal.

\section{Illustration ON AN ACtive Structure}

To demonstrate the two main claims of this paper, namely that MPC is a feasible and appealing alternative for active vibration suppression in the presence of actuator limits, the MPC strategy detailed in Section IV is applied to the cantilever beam apparatus illustrated in Figs. 2 and 3.
It is important to make our objective clear. We seek to minimize the displacement occurring at the beam tip while catering for actuator limits. However, since the tip displacement is not measured in practice, we instead minimize the estimate of tip velocity using the model obtained in Section V-B. This is achieved by designing an observer for the system state using the measured voltage across a piezoelectric patch (denoted $v$ in Fig. 3) and input $u$. This state is then used to predict the tip displacement (denoted $d$ in Fig. 3).

Using this prediction, we then solve the MPC optimization problem to determine a control action, which obeys the piezoelectric input actuator limits (associated with input signal $u$ in Fig. 3).

\section{A. Apparatus Description}

The experimental setup comprises a uniform aluminium beam, clamped at one end, and free at the other. Such an apparatus is a simple representation of many systems experienced in the field of active vibration control.

Although six piezoelectric transducers are bonded to the front and rear surfaces, only three patches are required in this application. Remaining patches are short circuited to minimize their influence on the structural dynamics.

The beam is $550 \mathrm{~mm}$ in length, $3 \mathrm{~mm}$ in thickness, and $50 \mathrm{~mm}$ in width. The transducer centers are mounted 55 and $215 \mathrm{~mm}$ from the clamped base. All transducers are manufactured from Physik Instrumente PIC151 piezoelectric ceramic and are $50 \mathrm{~mm}$ in length, $25 \mathrm{~mm}$ in width, and $0.25 \mathrm{~mm}$ in thickness.

The disturbance and control signals, $w$ and $u$, respectively, are applied through high-voltage amplifiers to the base patches. The location of these patches, over an area of high modal strain, affords sufficient authority over all structural modes. The mechanical strain at the beam center, acquired by buffering the induced open-circuit transducer voltage, is utilized as the feedback variable $v$. For the purpose of performance analysis, a Polytec Laser Vibrometer is employed to measure the tip displacement $d$.

\section{B. System Identification}

As pointed out in Section III, it is important to have a dynamic model of the system being controlled and we assume that the cantilever beam can be adequately modelled by the state-space structure (3) and (4).

Typically, however, the parameter values (i.e., all the matrix entries in $A, B, C, D)$ of such a model are not directly available. In such cases, system identification techniques are used to generate an estimate of the parameters from measurements of the input and/or output [26]. A detailed discussion of the many possible techniques is beyond the scope of this paper. Nevertheless, it is worth noting that if the available measurements are in the time domain, then it is standard practice to employ a maximum-likelihood approach [26], while if frequency domain measurements are available, then a frequency domain subspace method is typically used [29]. Both of these approaches are supported in the recent system identification package available at http://www.sigpromu.org/idtoolbox.

For the work presented here, frequency domain measurements were obtained at 908 nonequidistant frequencies ranging 

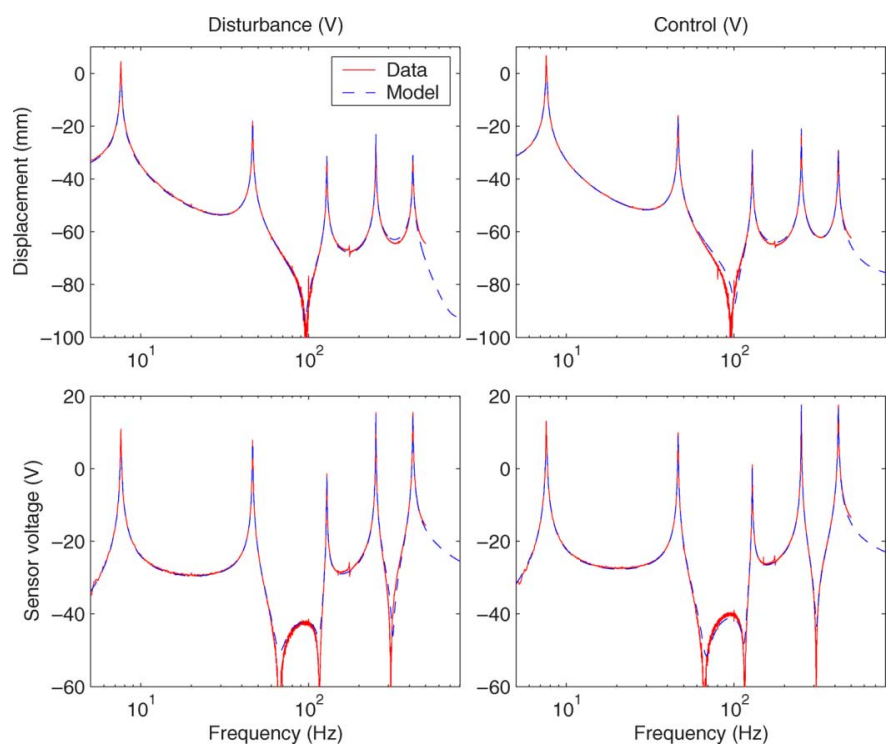

Fig. 4. Magnitude (dB) frequency response of identified beam model versus measured frequency response.

from 5 to $500 \mathrm{~Hz}$. The subspace algorithm described by [29] was then employed to obtain a two-input ( $u$ and $\omega$ ) two-output ( $d$ and $v$ ), 14th-order state-space model (which is adequate for modeling the system). While the apparatus has two available measurements (namely the voltage $v$ and tip displacement $d$ ), only the voltage $v$ is available for the purposes of control.

A satisfactory fit in the frequency domain can be observed in Fig. 4. The corresponding discrete-time state-space relationship between $u, \omega, d$, and $v$ is given by [note that the following has a more specific noise structure than (3) and (4)]:

$$
\begin{aligned}
& x_{t+1}=A x_{t}+B u_{t}+B_{\omega} \omega_{t} \\
& \underbrace{\left[\begin{array}{c}
v_{t} \\
d_{t}
\end{array}\right]}_{y_{t}}=\underbrace{\left[\begin{array}{c}
C_{v} \\
C_{d}
\end{array}\right]}_{C} x_{t}+\underbrace{\left[\begin{array}{c}
D_{v} \\
D_{d}
\end{array}\right]}_{D} u_{t}+\underbrace{\left[\begin{array}{c}
D_{\omega v} \\
D_{\omega d}
\end{array}\right]}_{D_{\omega}} \omega_{t}+\underbrace{\left[\begin{array}{c}
\tilde{e}_{t} \\
\bar{e}_{t}
\end{array}\right]}_{e_{t}} .
\end{aligned}
$$

The disturbance $\omega_{t}$ and measurement noises $\tilde{e}_{t}$ and $\bar{e}_{t}$ are assumed to be Gaussian distributed with zero mean and respective covariances of $\sigma_{\omega}$ and $\sigma_{v}$ and $\sigma_{d}$ with no cross-covariance, i.e.,

$$
\left[\begin{array}{c}
\omega_{t} \\
\tilde{e}_{t} \\
\bar{e}_{t}
\end{array}\right] \sim \mathcal{N}\left(\left[\begin{array}{l}
0 \\
0 \\
0
\end{array}\right],\left[\begin{array}{ccc}
\sigma_{\omega} & 0 & 0 \\
0 & \sigma_{v} & 0 \\
0 & 0 & \sigma_{d}
\end{array}\right]\right) .
$$

Since we cannot use the measured tip displacement for control purposes (since this is typically not available in practice), then the variance $\sigma_{d} \rightarrow \infty$ and for the purposes of estimating the state $d_{t}$ may be ignored. In addition, since we cannot measure the states directly, a Kalman Predictor is used to predict $\hat{x}_{t+1 \mid t}$. Combining these, we generate a Kalman gain matrix $L$ as given by (11) with

$$
\begin{aligned}
W & =B_{\omega} \sigma_{\omega} B_{\omega}^{T} \\
E & =\sigma_{v}+\sigma_{\omega} D_{\omega v}^{2} \\
S & =B_{\omega} \sigma_{\omega} D_{\omega v}
\end{aligned}
$$

such that

$$
\widehat{x}_{t+1 \mid t}=\left(A-L C_{v}\right) \widehat{x}_{t \mid t-1}+\left(B-L D_{v}\right) u_{t}+L v_{t} .
$$

\section{Handling Unmodeled High-Frequency Modes}

One important consideration for this application is that of unmodeled high frequency modes. Since the model does not include any modes above the fifth, then any high frequency control action could have a devastating effect on control performance (indeed this was observed during initial trials). To ameliorate this, we penalize high frequency control action in a standard manner by augmenting the plant model with a high-pass filter and include a penalty on this new signal, denoted $\bar{u}$, in the control objective function (see Section III).

More precisely, we constructed a fourth-order discrete-time Butterworth high-pass filter with 3-dB cutoff point at $450 \mathrm{~Hz}$ (c.f. frequency response in Fig. 4). The state-space matrices corresponding to this filter are denoted by $\left(A_{f}, B_{f}, C_{f}, D_{f}\right)$ and the augmented system is given by

$$
\begin{aligned}
{\left[\begin{array}{l}
x_{t+1} \\
\xi_{t+1}
\end{array}\right] } & =\left[\begin{array}{cc}
A & 0 \\
0 & A_{f}
\end{array}\right]\left[\begin{array}{l}
x_{t} \\
\xi_{t}
\end{array}\right]+\left[\begin{array}{c}
B \\
B_{f}
\end{array}\right] u_{t}+\left[\begin{array}{c}
B_{\omega} \\
0
\end{array}\right] \omega_{t} \\
{\left[\begin{array}{l}
y_{t} \\
\bar{u}_{t}
\end{array}\right] } & =\left[\begin{array}{cc}
C & 0 \\
0 & C_{f}
\end{array}\right]\left[\begin{array}{c}
x_{t} \\
\xi_{t}
\end{array}\right]+\left[\begin{array}{c}
D \\
D_{f}
\end{array}\right] u_{t}+\left[\begin{array}{c}
D_{\omega} \\
0
\end{array}\right] \omega_{t}+\left[\begin{array}{l}
I \\
0
\end{array}\right] e_{t} .
\end{aligned}
$$

Combining the beam model with the augmented filters results in an 18-state model. Note that the filtered states are known exactly and, therefore, the observer defined in (11), (16)-(18) does not need to be modified. However, since we are interested in using the filtered states as part of the control action, the augmented model (19), (20) must be used to form the optimization problem in (15).

\section{DSP Implementation}

As a further challenge to implementing MPC for fast systems, we wanted to ensure that the MPC algorithm, including state observer and quadratic programming solver, could be implemented on an inexpensive and standard digital signal processor (DSP) chip. In light of this, the particular hardware used for the MPC experiments is an Analog Devices ADSP-21262 [12] evaluation kit connected to an ancillary board containing a single channel analog-to-digital converter (ADC) and a single channel digital-to-analog converter (DAC).

The ADSP-21262 is a 32-bit floating point DSP running at a clock speed of $200 \mathrm{MHz}$. All instructions are single cycle (except division) with the capability of performing two instructions in parallel; although this feature is not being used in the current application. All software used for online purposes was developed manually in assembler in order to minimize overhead.

The offline calculations as shown in Procedure 1 were computed and the online calculations as shown in Algorithm IV.1 were executed on the DSP hardware according to the timing diagram in Fig. 5.

With regard to online calculations, there are two practical metrics commonly used for determining algorithm performance; the number of floating-point operations required and memory requirements. The former will be discussed in Section V-D.

The latter are shown in Table II. While it is difficult to comment on memory requirements more generally, we can afford to be critical about the current application. According to this 


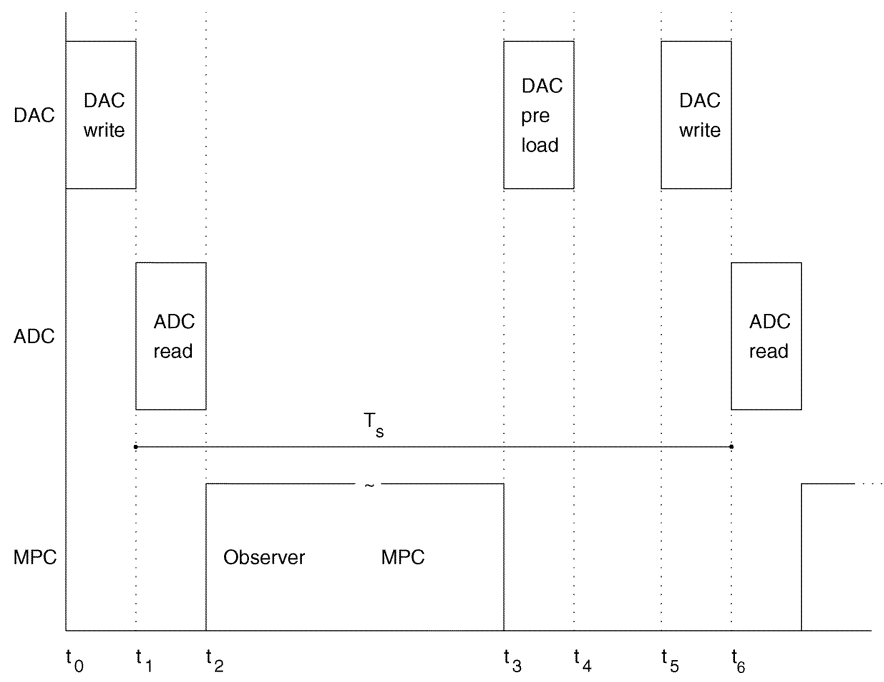

Fig. 5. Controller timing: the sample time $T_{s}$ is constant and $t_{1}$ and $t_{6}$ are the sampling "instants." $t_{0}$ (and $t_{5}$ ) mark the beginning of a DAC write operation, which incurs a known delay. Thus, we preempt the delay so that writing the input and reading the output occur within tolerable margins about $t_{1}$ (and $t_{6}$ ).

table the memory requirements for an 18th-order single-input single-output system with prediction horizon of 12 requires 1270 words, and at 32 bits/word that is approximately $10 \mathrm{~Kb}$ of total storage, which is very modest.

\section{EXPERIMENTAL RESULTS}

In this section, some experimental results are presented for the cantilever beam apparatus described in Section $\mathrm{V}$ and the MPC algorithm described in Sections III-V.

In order to adequately observe and control the high-frequency behavior of the output signal $y$, a sample rate of $5 \mathrm{kHz}$ (approximately 10 times the frequency of the highest mode under consideration) was tested.

In terms of the offline Procedure 1 [see also (16)-(18)], we use the following values. The noise covariances $\sigma_{\omega}$ and $\sigma_{v}$ are given by

$$
\sigma_{\omega}=1, \quad \sigma_{v}=10^{-12}
$$

The controller weighting matrices $Q$ and $R$ are set to

$$
Q=\left[\begin{array}{ccc}
0 & 0 & 0 \\
0 & 10 & 0 \\
0 & 0 & 100
\end{array}\right], \quad R=1 .
$$

Note that according to (20), the first diagonal term in $Q$ weights the measured voltage $v$, the second weights the estimated tip displacement $d$, while the third diagonal term weights the filtered input $\bar{u}$ (see Section V-C). This means that we are penalizing deviations in estimated tip displacement and also penalizing high frequency control action.

The constraint set $\mathbb{U}$ was constructed as

$$
\mathbb{U}=\left\{\mathbf{u} \in R^{N}:-0.2 \leq u_{i} \leq 0.2, \text { for } i=1, \ldots, N\right\}
$$

which represent simple bounds on actuator voltage prior to amplification (see Section V).
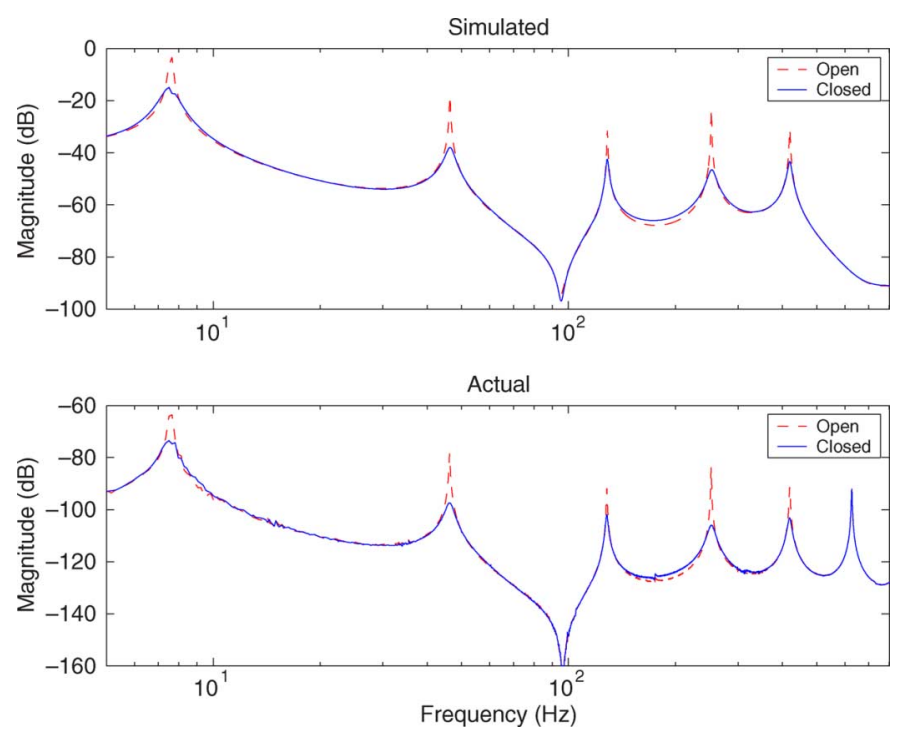

Fig. 6. Open- and closed-loop frequency responses (no actuator limits encountered).

\section{A. Controller Performance}

The first experiment is intended to show that the controller performs to a satisfactory level in the absence of hitting constraints. To achieve this, we used a periodic chirp disturbance ranging from 5 to $800 \mathrm{~Hz}$ and adjusted the disturbance gain so that actuator limits were not encountered. Fig. 6 shows the open- and closed-loop response for this controller in both simulation and in practice. It can be seen that the controller is performing well and the match between simulation and actual results is satisfactory.

In light of this, we proceeded to test the constraint handling capabilities of MPC by increasing the gain of the periodic chirp disturbance to ensure that limits were encountered.

In terms of comparing the performance, we also tested an LQG controller that is "saturated" when hitting constraints. In fact, the particular LQG controller gain matrix is given by (7) so that $u_{t+1}=K \widehat{x}_{t+1 \mid t}$ is the optimal control action in the absence of constraints. More precisely, the saturated LQG control law (SLQG) is given by

$$
u_{t+1}= \begin{cases}0.2, & \text { if } K \widehat{x}_{t+1 \mid t}>0.2 \\ -0.2, & \text { if } K \widehat{x}_{t+1 \mid t}<-0.2 \\ K \widehat{x}_{t+1 \mid t}, & \text { otherwise. }\end{cases}
$$

That is, if constraints are inactive (not encountered) then, by construction, the SLQG and MPC control laws coincide for any prediction horizon $N>0$ (see, e.g., [5]). Furthermore, even when hitting constraints, if the MPC prediction horizon is set to $N=1$, then SLQG and MPC still coincide [13]. For larger horizons this equivalence is not guaranteed.

Fig. 7 shows time-domain plots of the measured beam tip velocity when disturbed by a band-pass filtered step function under the control of MPC and SLQG at $5 \mathrm{kHz}$ sample rate with prediction horizon $N=12$. To generate the filtered step function an eighth-order Butterworth filter is used with pass band between $230-270 \mathrm{~Hz}$, which corresponds to the fourth mode (see Fig. 4). 

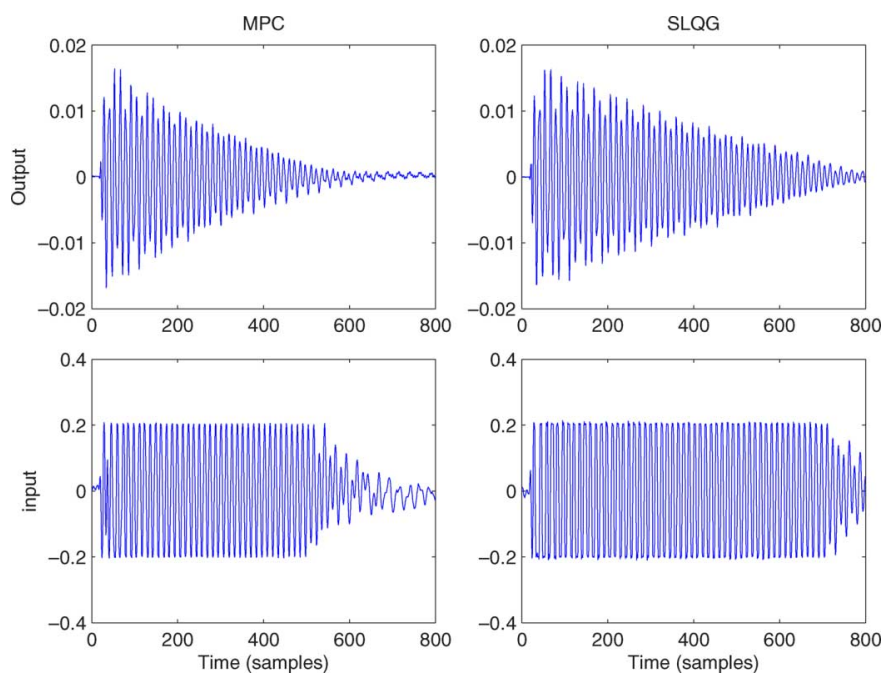

Fig. 7. Comparison of tip displacement for MPC and SLQG running at $5 \mathrm{kHz}$ sampling rate when a filtered step function (for fourth mode) is applied to the disturbance patch. Notice a distinct reduction in settling time. Note that input signals were measured and thus include some level of noise.
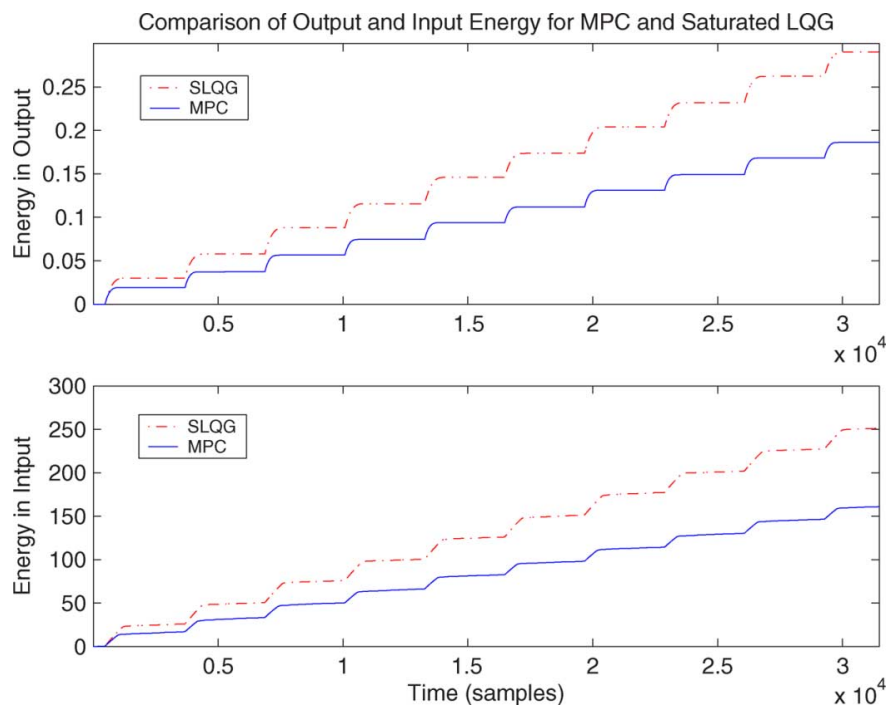

Fig. 8. Comparison of output and input energy for MPC and SLQG running at $5 \mathrm{kHz}$ sampling rate when a series of filtered step functions (for fourth mode) are applied to the disturbance patch. Notice that both in terms of input and output energy, MPC outperforms SLQG.

Although there is visible evidence from Fig. 7 that MPC outperforms SLQG, Fig. 8 confirms this by showing the output and input energy signals for a series of filtered step disturbances under both MPC and SLQG, where the energy signal of $y_{t}$, for example, is given by $\sum_{k=0}^{t}\left(y_{k}^{T} y_{k}\right)$.

\section{B. Empirical Limits for QP Solver}

As intimated in Section V-D, a common difficulty when using online optimization algorithms is uncertainty about solution time. Some methods offer better theoretical complexity limits than others (see, e.g., [45]), however, in practice the efficacy and efficiency of an algorithm often depends on the problem instance (see, e.g., [43]).

In order to provide some idea of the performance, we include a histogram of actual completion times for different horizon
TABLE III

Worst CASE TIME to Solve QP FOR DifFerent Horizons $N$

\begin{tabular}{|c|c|c|c|}
\hline $\mathrm{N}$ & QP clock cycles & Hit constraints & Max. time $(\mu \mathrm{s})$ \\
\hline 4 & 5962 & 74748 & 29.81 \\
\hline 6 & 10347 & 75609 & 51.735 \\
\hline 8 & 13745 & 75088 & 68.725 \\
\hline 10 & 20356 & 74379 & 101.78 \\
\hline 12 & 27742 & 75384 & 138.71 \\
\hline
\end{tabular}

TABLE IV

SPREAD OF QP SOLVE TIMES FOR $N=12$

\begin{tabular}{|c|c|}
\hline QP clock cycles & No. times QP solved \\
\hline $\mathrm{c}<5000$ & 1197566 \\
\hline $5000 \leq \mathrm{c}<7500$ & 90002 \\
\hline $7500 \leq \mathrm{c}<10000$ & 70154 \\
\hline $10000 \leq \mathrm{c}<12500$ & 48030 \\
\hline $12500 \leq \mathrm{c}<15000$ & 40836 \\
\hline $15000 \leq \mathrm{c}<17500$ & 32899 \\
\hline $17500 \leq \mathrm{c}<20000$ & 12444 \\
\hline $20000 \leq \mathrm{c}<22500$ & 4667 \\
\hline $22500 \leq \mathrm{c}<25000$ & 4923 \\
\hline $25000 \leq \mathrm{c}<27500$ & 534 \\
\hline $27500 \leq \mathrm{c}<30000$ & 5 \\
\hline $\mathrm{c}>30000$ & 0 \\
\hline
\end{tabular}

TABLE V

Number of CONSTRAints ACTIVE AT SOlution (LEFT COLUMN) AND Number of Times ACTIVE (Right COLUMN) FOR $N=12$

\begin{tabular}{|c|c|}
\hline No. active & No. times \\
\hline 1 & 106084 \\
\hline 2 & 114076 \\
\hline 3 & 55391 \\
\hline 4 & 23208 \\
\hline 5 & 5644 \\
\hline 6 & 68 \\
\hline $7-11$ & 0 \\
\hline 12 & 23 \\
\hline
\end{tabular}

lengths in Table III. We recorded the worst-case (i.e., maximum) time to solve the QP for different prediction horizons $N$ with sampling rate fixed at $5 \mathrm{kHz}$ and using a periodic chirp disturbance.

For each case, the controller is run for around $5 \mathrm{~min}$ (approximately 1500000 calls to the QP routine) and the maximum QP solve times are recorded. Also, recorded in Table III, are the number of times constraints were active at the solution.

Table IV provides some idea of the distribution for QP solve times with prediction horizon $N=12$. The disturbance signal applied is the same as for the previous test condition. Finally, Table V shows the number of constraints active at the solution of the QP versus the number of times each case occurred.

\section{CONCLUSION}

While MPC has attracted enormous research interest, it is commonly perceived that difficulties associated with the required online optimization limit its applicability. The empirical study of this issue has been a central motivation for this paper.

While we believe that the achieved active structural control results are of interest in their own right, the problem was also 
chosen for its suitability in examining these computational overhead issues.

In relation to this, a key outcome was to illustrate that using a very inexpensive hardware platform, the MPC action based on a nontrivial model (18 states), a reasonable prediction horizon (12 samples), and with input constraints, can be straightforwardly computed in less than $150 \mu \mathrm{s}$.

\section{ACKNOWLEDGMENT}

The authors would like to thank W. P. Heath for his help. His insight and suggestions were crucial to the positive outcome of this paper.

\section{REFERENCES}

[1] D. O. Anderson and J. B. Moore, Optimal Filtering. New York: Dover, 2005.

[2] J. Anthonis, J. Swevers, D. Moshou, and H. Ramon, " $H_{\infty}$-controller design for a vibrations isolating platform," Control Eng. Practice, vol. 7, no. 11, pp. 1333-1341, Nov. 1999.

[3] A. Bemporad, M. Morari, V. Dua, and E. N. Pistikopoulos, "The explicit linear quadratic regulator for constrained systems," Automatica, vol. 30, no. 1, pp. 3-20, Jan. 2002.

[4] A. Bemporad, M. Morari, V. Dua, and E. N. Pistikopoulos, "The explicit linear quadratic regulator for constrained systems," Automatica, vol. 38, no. 1, pp. 3-20, Jan. 2002.

[5] R. R. Bitmead, M. Gevers, and V. Wertz, Adaptive Optimal Control-The Thinking Man's GPC. Englewood Cliffs, NJ: Prentice-Hall, 1990.

[6] F. Borrelli, Constrained Optimal Control of Linear and Hybrid Systems. New York: Springer-Verlag, 2003, vol. 290.

[7] H. Chen and F. Allgöwer, "A quasi-infinite nonlinear model predictive control scheme with guaranteed stability," Automatica, vol. 34, no. 10, pp. $1205-1217,1998$.

[8] D. W. Clarke, C. Mohtadi, and P. S. Tuffs, "Generalized predictive control, parts 1 and 2," Automatica, vol. 23, no. 2, pp. 137-148, 1987.

[9] M. Dadfarnia, N. Jalili, Z. Liu, and D. M. Dawson, "An observer-based piezoelectric control of flexible cartesian robot arms: Theory and experiment," Control Eng. Practice, vol. 12, no. 8, pp. 1041-1053, Aug. 2004.

[10] S. Daley, F. A. Johnson, J. B. Pearson, and R. Dixon, "Active vibration control for marine applications," Control Eng. Practice, vol. 12, no. 4, pp. 465-474, Apr. 2004.

[11] A. J. den Hamer, G. Z. Angelis, and N. B. Roozen, "Broad-band active vibration suppression using PPF focused on industrial application," IEEE/ASME Trans. Mechatronics, vol. 10, no. 2, pp. 146-153, Apr. 2005.

[12] Analog Devices, "Evaluation kit for ADSP-21262 SHARC processor," 1995-2005.

[13] J. De Dona, G. Goodwin, and M. Seron, "Anti-windup and model predictive control: Reflections and connections," Euro. J. Control, vol. 6, no. 5, pp. 467-477, 2000.

[14] J. L. Fanson and T. K. Caughey, "Positive position feedback-control for large space structures," AIAA J., vol. 28, no. 4, pp. 717-724, Apr. 1990.

[15] A. V. Fiacco, Introduction to Sensitivity and Stability Analysis in Nonlinear Programming. London, U.K.: Academic, 1983.

[16] C. R. Fuller, S. J. Elliott, and P. A. Nelson, Active Control of Vibration. London, U.K.: Academic, 1996.

[17] C. E. Garcia, D. M. Prett, and M. Morari, "Model predictive control: Theory and practice-A survey," Automatica, vol. 25, no. 3, pp. 335-348, 1989.

[18] C. J. Goh and T. K. Caughey, "On the stability problem caused by finite actuator dynamics in the collocated control of large space structures," Int. J. Control, vol. 41, no. 3, pp. 787-802, 1985.

[19] T. Goh, Z. Li, B. Chen, T. Lee, and T. Huang, "Design and implementation of a hard disk drive servo system using robust and perfect tracking approach," IEEE Trans. Control Syst. Technol., vol. 9, no. 2, pp. 221-233, Mar. 2001.
[20] D. Goldfarb and A. Idnani, "A numerically stable dual method for solving strictly convex quadratic programs," Math. Program., vol. 27, pp. 1-33, 1983

[21] G. H. Golub and C. F. Van Loan, Matrix Computations, Third Edition. Baltimore, MD: The Johns Hopkins Univ. Press, 1996.

[22] G. Guo, D. Wu, and T. Chong, "Modified dual-stage controller for dealing with secondary-stage actuator satuation," IEEE Trans. Magn., vol. 39, no. 6, pp. 3587-3592, Nov. 2003.

[23] M. A. Hopkins, D. A. Henderson, R. W. Moses, T. Ryall, D. G. Zimcik, and R. L. Spangler, "Active vibration-suppression systems applied to twin-tail buffeting," in Proc. SPIE Smart Structures Mater.: Ind. Commer. Appl. Smart Structures Technol., 1998, pp. 27-33.

[24] D. J. Inman, Engineering Vibration, 2nd ed. Englewood Cliffs, NJ: Prentice-Hall, 2001.

[25] T. A. Johansen, W. Jackson, R. Schreiber, and P. Tondel, "Hardware synthesis of explicit model predictive controllers," IEEE Trans. Control Syst. Technol., vol. 15, no. 1, pp. 191-197, Jan. 2007.

[26] L. Ljung, System Identification: Theory for the User-Second Edition. Englewood Cliffs, NJ: Prentice-Hall, 1999.

[27] J. M. Maciejowski, Predictive Control with Constraints. Essex, U.K.: Pearson Education, 2002.

[28] D. Q. Mayne, J. B. Rawlings, C. V. Rao, and P. O. M. Scokaert, "Constrained model predictive control: Stability and optimality," Automatica, vol. 36, pp. 789-814, 2000.

[29] T. McKelvey, H. Akcay, and L. Ljung, "Subspace based multivariable system identification from frequency response data," IEEE Trans. Autom. Control, vol. 41, no. 7, pp. 960-978, Jul. 1996.

[30] Z. Mohamed, J. M. Martins, M. O. Tokhi, J. Sa da Costa, and M. A. Botto, "Vibration control of a very flexible manipulator system," Control Eng. Practice, vol. 13, no. 3, pp. 267-277, Mar. 2005.

[31] S. O. R. Moheimani, D. Halim, and A. J. Fleming, Spatial Control of Vibration: Theory and Experiments. Singapore: World Scientific, 2003.

[32] S. O. R. Moheimani, B. J. G. Vautier, and B. Bhikkaji, "Experimental implementation of extended multivariable PPF control on an active structure," IEEE Trans. Control Syst. Technol., vol. 14, no. 3, pp. 443-445, May 2006.

[33] M. L. Munjal, Acoustics of Ducts and Mufflers. New York: WileyInterscience, 1987.

[34] K. R. Muske and J. B. Rawlings, "Model predictive control with linear models," AIChE J., vol. 39, no. 2, pp. 262-287, 1993.

[35] P. A. Nelson and S. J. Elliott, Active Control of Sound. London, U.K.: Academic, 1992.

[36] K. Peng, B. Chen, T. Lee, and V. Venkataramanan, "Design and implementation of a dual-stage HDD servo system via composite nonlinear control approach," Mechatron., vol. 14, pp. 965-988, 2004.

[37] I. R. Petersen and H. R. Pota, "Minimax LQG optimal control of a flexible beam," Control Eng. Practice, vol. 11, no. 11, pp. 1273-1287, Nov. 2003.

[38] M. J. D. Powell, "On the quadratic programming Algorithm of Goldfarb and Idnani," Math. Program. Study, vol. 25, pp. 46-61, 1985.

[39] S. J. Qin and T. A. Badgewell, "A survey of industrial model predictive control technology," Control Eng. Practice, vol. 11, pp. 733-764, 2003.

[40] K. Schittkowski, "QL: A fortran code for convex quadratic programming-Users's guide," Dept. Math., Univ. Bayreuth, Bayreuth, Germany, 2003.

[41] M. M. Seron, G. C. Goodwin, and J. De Dona, "Characterizations of receding horizon control for constrained linear systems," Asian J. Control, vol. 5, no. 2, pp. 271-286, 2003.

[42] D. Sun and J. Mills, "Combined PD feedback and distributed piezoelectric-polymer vibration control of a single-link flexible manipulator," in Proc. IEEE/IRSI Int. Conf. Intell. Robot. Syst., 1998, pp. 667-672.

[43] M. J. Todd, "The many facets of linear programming," Ithaca, NY, Tech. Rep. 14853, 2000.

[44] P. Tøndel, T. A. Johansen, and A. Bemporad, "An algorithm for multiparametric quadratic programming and explicit MPC solutions," Austomatica, vol. 39, no. 3, pp. 489-497, Mar. 2003.

[45] A. G. Wills and W. P. Heath, "EE03016-Interior-point methods for linear model predictive control," Sch. Electr. Eng. Comput. Sci., Univ. Newcastle, Upon Tyne, Australia, 2003.

[46] E. Zafiriou, "Robust model predictive control of processes with hard constraints," Comput. Chem. Eng., vol. 14, no. 4-5, pp. 359-371, May 1990. 


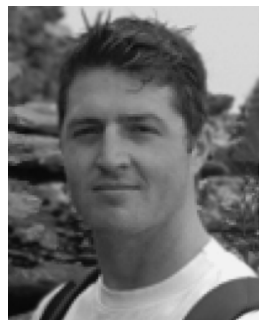

Adrian G. Wills was born in Orange, N.S.W. Australia. He received the B.E. (elec.) and the Ph.D. degrees from The University of Newcastle, Callaghan, Australia, in 1999 and 2003, respectively.

Since then he has held a Postdoctoral Research Position with the School of Electrical Engineering and Computer Science, The University of Newcastle, where his research has been focused on the area of system identification.

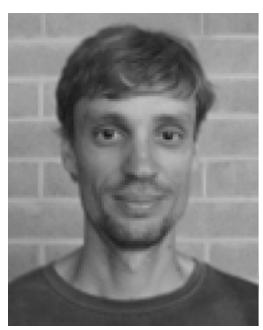

Dale Bates (S'07) was born in Castlemaine, VIC, Australia. He received the B.E. degree in computer engineering from the University of Newcastle, Callaghan, Australia, in 2004, where he is currently pursuing the Ph.D. degree under the guidance of Prof. B. Ninness from the University of Newcastle.

His reseach interests include control and MIMO wireless communication systems.

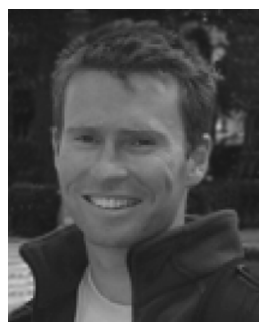

Andrew J. Fleming (M'03) was born in Dingwall, Scotland, in 1977. He received the B.S. degree in electrical engineering and the $\mathrm{Ph} . \mathrm{D}$ degree from The University of Newcastle, Callaghan, Australia, in 2000 and 2004.

Currently, he is an Australian Research Council fellow with the School of Electrical Engineering and Computer Science, The University of Newcastle. In 2005, he was a research academic with the Centre for Complex Dynamics and Control, The University of Newcastle. His research interests include micro-cantilever sensors, nano-positioning, and sensor-less control of sound and vibration.

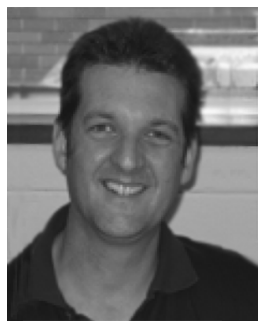

Brett Ninness (M'93) was born in 1963, in Singleton, Australia. He received the B.E., M.E., and $\mathrm{Ph} . \mathrm{D}$. degrees in electrical engineering from the University of Newcastle, Australia, in 1986, 1991, and 1994, respectively.

Since 1993, he has been with the School of Electrical Engineering and Computer Science, The University of Newcastle, where he is currently a Professor. His research interests include the areas of system identification and stochastic signal processing, in which he has authored approximately 100 papers in journals and conference proceedings. He has served on the editorial board of the IEEE TRANSACTIONS ON AUTOMATIC CONTROL.

Prof. Ninness has served on the editorial board of Automatica and is currently the Editor-In-Chief of the Control Theory and Applications. Together with $\mathrm{H}$. Hjalmarsson, he jointly organized the 14th IFAC Symposium on System Identification in Newcastle, Australia, in 2006. Further details of his professional activities are available at http://www.sigpromu.org/brett.

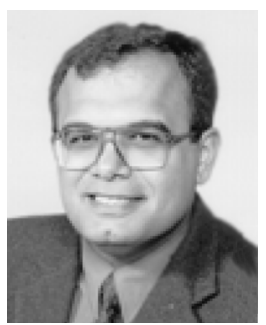

S. O. Reza Moheimani (SM'00) received the Ph.D. degree in electrical and electronics engineering from University of New South Wales, Australian Defence Force Academy, Canberra, Australia, in 1996.

Following a research position at the same institution, he joined the University of Newcastle in 1997, where he is currently an Associate Professor in the School of Electrical Engineering and Computer Science, the Assistant Dean Research (Engineering), the head of the Laboratory for Dynamics and Control of NanoSystems, and an Associate Director of the ARC Centre for Complex Dynamic Systems and Control, an Australian Government Centre of Excellence. He is an Associate Editor for the IEEE TRANSACTIONS ON CONTROL SYSTEMS TECHNOLOGY.

Dr. Moheimani is an Associate Editor of several international journals and has chaired a number of international workshops and conferences. He has published two books, several edited volumes, and over 150 refereed articles in the areas of robust control and estimation, smart structures, active noise and vibration control and mechatronic systems and nanotechnology. 